\title{
Frecuencia y severidad de hipomineralización incisivo molar (HIM) en un grupo de niños mexicanos, 2014
}

José Francisco Murrieta-Pruneda ${ }^{\star}, \mathrm{PhD}_{1}$, Jessica Torres-Vargas, $\mathrm{OD}_{1}$, Julieta del Carmen Sánchez-Meza, Esp.

I Facultad de Estudios Superiores Zaragoza, Universidad Nacional Autónoma de México

${ }_{2}$ Universidad Autónoma Metropolitana Хоснімilco, México D. F., México

\section{Recibido: 28 de enero del 2016 Aprobado: 30 de marzo del 2016}

*Autor de correspondencia: José Francisco Murrieta-Pruneda. División de Estudios de Posgrado e Investigación, Facultad de Estudios Superiores Zaragoza, Universidad Nacional Autónoma de México. Batalla 5 de Mayo sN, Ejército Oriente, Iztapalapa, 09230 Ciudad de México, México. Teléfono: (55)56230701. Correo electrónico: francisco.murrieta@gmail.com

Cómo citar este artículo: Murrieta-Pruneda JF, Torres-Vargas J, Sánchez-Mesa JDC. Frecuencia y severidad de hipomineralización incisivo molar (HIM) en un grupo de niños mexicanos, 2014. Rev Nac Odontol. 2016;12(23):7-14. doi: 10.16925/od.v12i23.1377

Resumen. Introducción: la frecuencia de la hipomineralización incisivo molar (HIM) es variable y puede estar presente hasta en el $25 \%$ de la población. Objetivo: establecer la frecuencia y la severidad de нiм en un grupo de niños mexicanos. Materiales y métodos: el estudio fue de carácter transversal; se examinó una muestra por conveniencia integrada por 433 escolares de ambos sexos entre 8 y 12 años de edad. La encuesta consideró la exploración clínica de los primeros molares e incisivos permanentes considerando los criterios establecidos por la Academia Europea de Odontología Pediátrica (EAPD), y para la severidad, los criterios de Mathu-Muju y Wright. Para el levantamiento epidemiológico, previamente se estandarizó a una examinadora (kappa =0,93). Resultados: el 13,9\% de los escolares presentó HIM, siendo más frecuente en niños $(8,1 \%)$ que en niñas $(5,8 \%)$, asociándose con esta variable $(\mathrm{p}=0,049)$. Por edad, las categorías de 8 y 10 años fueron las que presentaron un mayor número de casos sin mostrar relevancia estadística con el evento de estudio. Los primeros molares resultaron estar más involucrados en los patrones de HIM, y asimismo, en cuanto a la severidad del HIM, la condición leve fue la más frecuente. Conclusiones: La frecuencia y la severidad observadas muestran un panorama que requiere llevar a cabo el seguimiento de los niños con HIM dado el riesgo que representan para el establecimiento de caries dental.

Palabras clave: alteraciones dentales, escolares, estudio transversal, hipomineralización incisivo-molar. 


\title{
Frequency and Severity of Molar Incisor HypoMineralization (HIM) in a group of Mexican children, 2014
}

\begin{abstract}
Introduction: The frequency of Molar Incisor Hypomineralization (HIM) is variable and may be present in as many as $25 \%$ of the population. Objective: To determine the frequency and severity of HIM in a group of Mexican children. Materials and Methods: The study was cross-sectional; a sample was examined through integrated convenience in 433 students of both genders between 8 and 12 years old. The survey included clinical exploration of the first permanent molar and incisive while taking into account the criteria established by the European Academy of Paediatric Dentistry (EAPD), and for the severity, the criteria of Mathu-Muju and Wright. For the epidemiological lifting, an examiner was previously standardized (kappa $=0.93$ ). Results: $13.9 \%$ of the students presented HIM, condition more frequently seen in boys $(8.1 \%)$ than in girls $(5.8 \%)$, associated to this variable $(\mathrm{p}=0.049)$. Age-specific, the categories of 8 and 10 year olds presented the highest number of cases without statistical relevance with the event of this study. The first molars resulted to be more involved in HIM patterns, and likewise, as of the severity of HIM, the minor condition was more frequent. Conclusion: The frequency and severity reported indicate a scenario that requires follow-up of children affected by HIM, given the risk of permanent tooth decay.
\end{abstract}

Keywords: teeth disorder, schoolchildren, cross-sectional study, hypomineralization, molar incisive.

\section{Frequência e severidade de hipomineralização Molar- Incisivo (HMI) em um grupo de crianças mexicanas, 2014}

Resumo. Introdução: a frequência da hipomineralização Molar-Incisivo (HMI) é variável e pode estar presente até em $25 \%$ da população. Objetivo: estabelecer a frequência e a severidade de HIM em um grupo de crianças mexicanas. Materiais e métodos: o estudo foi de carácter transversal; examinou-se uma amostra por conveniência integrada por 433 escolares de ambos os sexos entre 8 e 12 anos de idade. A pesquisa considerou a exploração clínica dos primeiros molares e incisivos permanentes considerando os critérios estabelecidos pela Academia Europeia de Odontologia Pediátrica (EAPD), e para a severidade, os critérios de Mathu-Muju e Wright. Para o levantamento epidemiológico, previamente foi padronizada a uma examinadora (kappa $=0,93$ ). Resultados: 13,9\% dos escolares apresentou HIM, sendo mais frequente em meninos $(8,1 \%)$ do que em meninas (5,8\%), associando-se com esta variável $(\mathrm{p}=0,049)$. Por idade, as categorias de 8 e 10 anos foram as que apresentaram um maior número de casos sem mostrar relevância estatística com o evento de estudo. Os primeiros molares estiveram mais envolvidos nos padrões de HMI, e igualmente, em relação à severidade do HMI, a condição leve foi a mais frequente. Conclusões: A frequência e a severidade observadas evidenciam um cenário que exige executar o acompanhamento das crianças com HMI dado o risco que representam para o estabelecimento de cárie dentaria.

Palavras chave: alterações dentarias, escolares, estudo transversal, hipomineralização molar-incisivo. 


\section{Introducción}

La Academia Europea de Odontología Pediátrica (EAPD) define el término hipomineralización incisivo molar (HIM) [1] para caracterizar a esta entidad clínica que afecta a los primeros molares e incisivos de la segunda dentición, comprometidos durante el periodo de amelogénesis [1-16]. Su importancia se debe a que afecta a los dientes presentes con la pérdida de esmalte poseruptiva, lo que constituye un riesgo para el desarrollo de lesiones cariosas de mayor severidad $[17,18]$.

La incidencia de caries se observa con más frecuencia en dientes hipomineralizados, los cuales se distinguen por la presencia de opacidades de color amarillo o marrón. Tal criterio permite al estomatólogo definir el tratamiento más adecuado, basado en el riesgo que tienen los niños con HIM para desarrollar caries dental [19-22]. Esta alteración es considerada un problema de salud pública, pues según los resultados reportados, al menos uno de cada cinco niños la tiene; no obstante, debido a la baja prioridad que se le ha dado en los programas asistenciales públicos, los padres de niños con HIM centran la mirada en la práctica privada como alternativa para resolver este problema [4-10].

La etiología exacta no se ha definido en su totalidad, pero factores como la desnutrición y la fiebre asociadas a enfermedades infecciosas, han dado lugar a la posibilidad de considerar que los dientes con HIM pueden estar vinculados a la salud de la madre durante el embarazo, y en el caso de los niños, a los antecedentes de fiebre alta, traumáticos, de hipoxia y de algún estado nutricional carencial $[4,9,23,24]$. Sin embargo, hasta la fecha no hay pruebas suficientemente claras que lleven a afirmar que estos factores son determinantes para el establecimiento de HIM $[4-7,10]$.

Desde un punto de vista epidemiológico, la frecuencia de HIM es variable dado que se ha observado en el 4,6\% de niños alemanes [25] y en el $27,7 \%$ de niños tailandeses [23]. Infortunadamente, la mayoría de los estudios de frecuencia de HIM se han realizado en países europeos y asiáticos [2633 ], lo que no ha dejado precisar qué tan parecido o diferente es el comportamiento de esta alteración en niños de países latinoamericanos $[9,18,20,21$, 34, 35]. En términos clínicos, los primeros molares permanentes se ven afectados con mayor frecuencia que los incisivos $[7,17,19,21,22]$, y en términos de severidad, la condición leve es la más común [7,
$16,17,22,25,26]$. El objetivo de este estudio fue establecer la frecuencia y la severidad de HIM por edad y sexo en 433 niños en edad escolar.

\section{Materiales y métodos}

Se llevó a cabo un estudio transversal en el cual se examinó una muestra por conveniencia integrada por 433 escolares entre 8 y 12 años de edad, de la zona oriente de la Ciudad de México, con una edad promedio de 9,8 años (DE 1,3). El proyecto fue aprobado por el Comité de Bioética y Bioseguridad de la Línea de Investigación en Oclusión Dental (LI-FESZ-230506) de la Facultad de Estudios Superiores Zaragoza de la unAM. Así mismo, se solicitó a los padres que firmaran el consentimiento informado autorizando la inclusión de sus hijos en el estudio, cubriendo así los aspectos ético-legales de la investigación.

Fueron excluidos del estudio 28 escolares debido a alguna de las siguientes razones: niños cuyos padres no autorizaron su inclusión en el estudio, que tenían pérdida prematura de los dientes de interés para el estudio por razones diferentes a la presencia de HIM, que estaban en tratamiento de ortodoncia, que no cooperaron al momento del examen o que tenían alguna discapacidad física.

La encuesta epidemiológica consistió en la exploración bucal de los niños con el examen de los primeros molares y los incisivos secundarios bajo condiciones húmedas de las superficies dentarias, considerando los criterios establecidos por la Academia Europea de Odontología Pediátrica [1] para valorar la ausencia o presencia de opacidades bien demarcadas, fractura de esmalte poseruptiva, restauraciones atípicas o extracciones de molares debido a HIM.

De igual modo, se clasificó cualquier caso positivo de HIM de acuerdo con los siguientes patrones: tipo I cuando perjudicó solamente a los molares; tipo II cuando los molares y los incisivos estaban afectados; y tipo III cuando la evidencia del HIM estaba tanto en molares e incisivos superiores como inferiores. Se emplearon los criterios de Mathu-Muju y Wright [22] para valorar la severidad de la hipomineralización de acuerdo con su condición, ya fuera leve, moderada o severa. Para el examen bucal, una cirujana dentista fue previamente estandarizada (kappa $=0,93$ ).

Los datos fueron tabulados en una hoja de cálculo de Microsoft Excel Office $2010^{\circledR}$, la cual 
permitió exportarlos al paquete estadístico sPSS $\mathrm{IBM}^{\circledR}$ versión 2.0 (Statistical Package for the Social Sciences, Nueva York, Estados Unidos).

A fin de evaluar el comportamiento de la frecuencia y severidad de HIM, se calcularon frecuencias absolutas y relativas. Para revisar la posible relación de la frecuencia de HIM (por patrón y por severidad) con la edad, se calculó el valor de Chi-cuadrado de Pearson, y cuando alguna de las celdas tuviera un valor igual o menor a cinco, se calculó el valor de razón de verosimilitud.

Para evaluar la posible relación de la frecuencia del HIM con el sexo, se calculó el valor de Chi-cuadrado de Mantel y Haenszel, debido a que las variables en cuestión eran dicotómicas con representación de categorías nominales, cuya distribución se presentó en tablas de contingencia de 2x2 (tablas tetrateóricas), en las cuales los valores esperados en cada celda siempre fueron mayores a cinco, y se calcularon los estadísticos de Breslow-Day y de Tarone para el contraste de la hipótesis de homogeneidad.

\section{Resultados}

El promedio de edad de los participantes en el estudio fue de 9,8 años ( $D E \pm 1,3$ ), y de los escolares evaluados participaron 198 niñas $(45,7 \%)$ y 235 niños $(54,3 \%)$. En relación con la edad, las categorías de 7,8 y 9 años tuvieron mayor representatividad en la muestra $(21,2 \%, 22,4 \%$ y $22,4 \%$, respectivamente).

La hipomineralización incisivo molar se presentó en el $14,0 \%$ de la población. De acuerdo con la edad, las categorías de 8 y 10 años fueron las que tuvieron un mayor número de casos de нім $(3,9 \%$ y $3,5 \%)$, en comparación con el resto de las categorías cuyos porcentajes fueron menores, en un rango entre 1,2 y $2,8 \%$, diferencias que no resultaron ser relevantes (razón de verosimilitud $=5,517$, $\mathrm{p}=0,238$ ). En cuanto al sexo, se observó mayor frecuencia de casos de HIM en niños que en niñas $(8,1 \%$ y $5,8 \%$, respectivamente), $(\mathrm{x} 2 \mathrm{MH}=3,881$, $\mathrm{p}=0,049)$, comportamiento que resultó ser estadísticamente significativo (tabla 1).

En relación con el porcentaje de dientes involucrados con HIM, se observó que los primeros molares estuvieron afectados con más frecuencia que los incisivos, puesto que el rango de frecuencia en molares fue desde $6,0 \%$ hasta $13,9 \%$, mientras que en incisivos osciló entre $1,2 \%$ y $3,5 \%$, diferencias que resultaron
Tabla 1. Distribución porcentual de casos de Him de acuerdo con la edad y el sexo

\begin{tabular}{|c|c|c|c|c|c|}
\hline & \multicolumn{2}{|c|}{ Ausente } & \multicolumn{2}{|c|}{ Presente } & \multirow[b]{2}{*}{ n } \\
\hline & f & $\%$ & $\mathrm{f}$ & $\%$ & \\
\hline \multicolumn{6}{|l|}{ Edad } \\
\hline 8 & 77 & 17,8 & 15 & 3,5 & 92 \\
\hline 9 & 86 & 19,9 & 11 & 2,5 & 97 \\
\hline 10 & 80 & 18,5 & 17 & 3,9 & 97 \\
\hline 11 & 66 & 15,2 & 5 & 1,2 & 71 \\
\hline \multirow[t]{2}{*}{12} & 64 & 14,8 & 12 & 2,8 & 76 \\
\hline & & & & \multicolumn{2}{|c|}{$\mathrm{p}=0,238$} \\
\hline \multicolumn{6}{|l|}{ Sexo } \\
\hline Niños & 163 & 37,6 & 35 & 8,1 & 198 \\
\hline \multirow[t]{2}{*}{ Niñas } & 210 & 48,5 & 25 & 5,8 & 235 \\
\hline & & & & \multicolumn{2}{|c|}{$\mathrm{p}=0,049$} \\
\hline Total & 373 & 86,1 & 60 & 13,9 & 433 \\
\hline
\end{tabular}

Fuente: elaboración propia

ser altamente significativas $(\mathrm{x} 2 \mathrm{MH}=44,381, \mathrm{p}=0,0001$; véase figura 1).

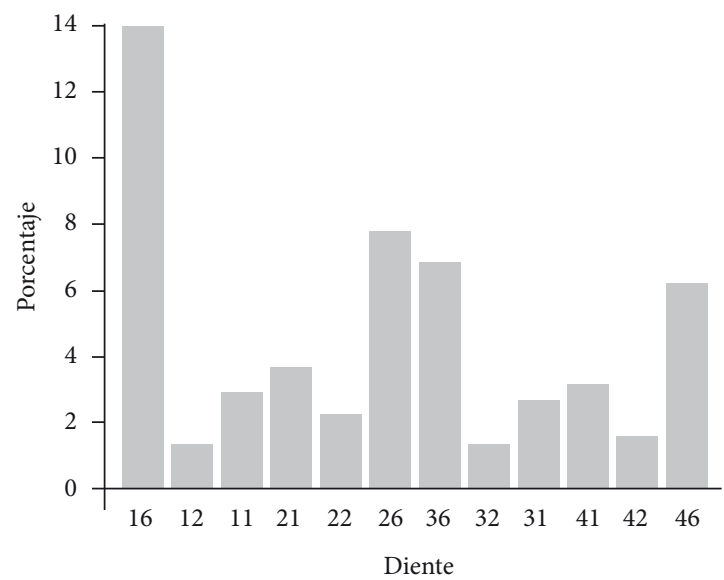

Figura 1. Distribución porcentual de acuerdo con los dientes involucrados en los casos de нiм observados

Fuente: elaboración propia

Según el patrón de los casos de HIM detectados, se observó que cerca de tres de cada cuatro casos correspondieron al patrón tipo I, en el cual solo estuvieron involucrados los primeros molares permanentes (73\%), el tipo II fue el segundo más frecuente $(23 \%)$ y el tipo in fue el menos frecuente (4\%; véase figura 2 ). 


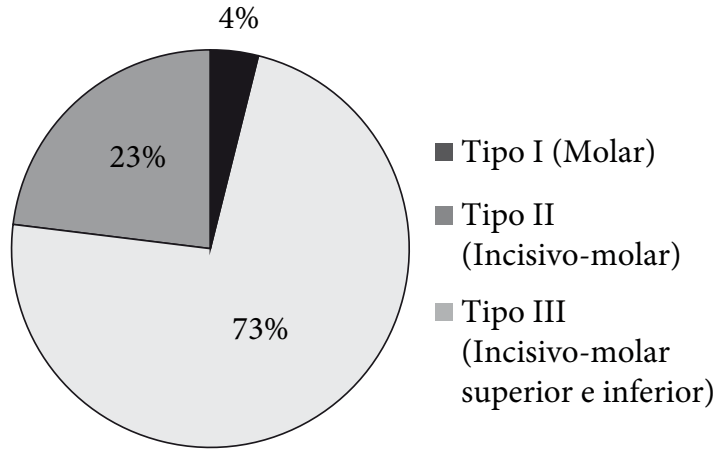

Figura 2. Distribución porcentual de Hiм según el patrón de comportamiento

Fuente: elaboración propia
La distribución porcentual de casos de HIM según su severidad por edad fue relativamente proporcional en cada una de las categorías, valores cuyos rangos estuvieron entre $0,7 \%$ y $1,0 \%$ en la condición leve, entre $0,5 \%$ y $2,1 \%$ en la condición moderada y entre $0,0 \%$ y $0,5 \%$ en la condición severa, comportamiento que no mostró relación alguna entre estas dos variables (razón de verosimilitud $=15,138, \mathrm{p}=0,234$ ).

En cuanto al sexo, las frecuencias relativas observadas se comportaron de la siguiente manera: $3,0 \%$ para niñas y 3,9\% para niños en la condición leve; $2,3 \%$ para niñas y $3,2 \%$ para niños en la condición moderada; y 0,5\% para niñas y $0,9 \%$ para niños en la condición severa, comportamiento que tampoco mostró asociación alguna entre estas dos variables (razón de verosimilitud $=4,657, \mathrm{p}=0,199$; tabla 2).

Tabla 2. Distribución de casos de Him según su severidad por edad y sexo

\begin{tabular}{|c|c|c|c|c|c|c|c|c|c|}
\hline & \multicolumn{2}{|c|}{ Ausente } & \multicolumn{2}{|c|}{ Ligera } & \multicolumn{2}{|c|}{ Moderada } & \multicolumn{2}{|c|}{ Severa } & \multirow[b]{2}{*}{$\mathbf{n}$} \\
\hline & f & $\%$ & f & $\%$ & $\mathrm{f}$ & $\%$ & $\mathrm{f}$ & $\%$ & \\
\hline \multicolumn{10}{|l|}{ Edad } \\
\hline 8 & 77 & 17,8 & 6 & 1,4 & 9 & 2,1 & 0 & 0,0 & 92 \\
\hline 9 & 86 & 19,9 & 5 & 1,2 & 4 & 0,9 & 2 & 0,5 & 97 \\
\hline 10 & 80 & 18,5 & 8 & 1,8 & 7 & 1,6 & 2 & 0,5 & 97 \\
\hline 11 & 66 & 15,2 & 3 & 0,7 & 2 & 0,5 & 0 & 0,0 & 71 \\
\hline \multirow[t]{2}{*}{12} & 64 & 14,8 & 8 & 1,8 & 2 & 0,5 & 2 & 0,5 & 76 \\
\hline & & & & & & & & \multicolumn{2}{|c|}{$\mathrm{p}=0,234$} \\
\hline \multicolumn{10}{|l|}{ Sexo } \\
\hline Niños & 163 & 37,6 & 17 & 3,9 & 14 & 3,2 & 4 & 0,9 & 198 \\
\hline \multirow[t]{2}{*}{ Niñas } & 210 & 48,5 & 13 & 3,0 & 10 & 2,3 & 2 & 0,5 & 235 \\
\hline & & & & & & & & \multicolumn{2}{|c|}{$\mathrm{p}=0,199$} \\
\hline Total & 373 & 86,1 & 30 & 6,9 & 24 & 5,5 & 6 & 1,4 & 433 \\
\hline
\end{tabular}

$\mathrm{f}:$

n:

Fuente: elaboración propia

\section{Discusión}

La frecuencia de HIM en la población de estudio tuvo un comportamiento similar a lo reportado por López [34] en argentinos y uruguayos, por Bhaskar [19] en indios, por Janković [15] en bosnios, por Petrou [25] en alemanes, por Tariq [8] en emiratíes y por Jans [21] en chilenos, entre otros $[9-11,19,20,25,27,28,30]$, pero inferior a lo obser- vado por Souza [9] y Da Costa [18] en brasileños, por Ghanim [27] en iraquíes, por Mahoney [29] en neozelandeses y por Grošelj [17] en slovenios, lo cual puso en evidencia la variabilidad de su frecuencia. No se observó asociación de HIM con la edad. El único referente distinto es el observado por Parikh [28], quien estableció que la frecuencia y la severidad de HIM se incrementaron de acuerdo con la edad. 
En cuanto a los dientes involucrados en esta alteración, los primeros molares fueron los más afectados, comportamiento similar a los reportados por Kellerhorff [12], Souza [9], Ghanim [27], Parikh [28], Gotler [14] y Jans [21], los cuales pudieron verse afectados en su desarrollo durante los primeros años de vida, que es cuando se completa la calcificación de los primeros molares secundarios. Por esta razón, la investigación relacionada con la etiología de HIM se ha centrado en los acontecimientos ambientales que ocurren en el último trimestre de gestación y en los primeros años de vida del niño $[16,27]$, dado que representan un riesgo más alto para que tenga lugar la hipomineralización $[25,33]$.

El patrón i fue más frecuente, pues en la mayoría de los casos se observó que solo los primeros molares secundarios estaban afectados [16]. Sin embargo, como establecieron Kellerhoff [12] y Álvarez [35], una lesión provocada por la hipomineralización del esmalte, de origen sistémico y diverso, que afecta a los primeros molares permanentes, con frecuencia se asocia a lesiones en los incisivos superiores e inferiores permanentes, condición que solo fue observada en uno de cada cuatro casos.

En cuanto a la severidad de HIM, los casos relacionados con las condiciones leve y moderada fueron los más frecuentes, comportamiento similar a lo observado por Petrou [25], Ghanim [27], Allazzam [32] y López [34]. La severidad de нiм no resultó estar asociada con la edad o el sexo, comportamiento que coincide con lo reportado por Petrou [25], Ghanim [27], Allazzam [32] y López [34]; este comportamiento no resulta ser tan desfavorable al considerar el riesgo para desarrollar caries dental en comparación con los casos de HIM moderada y severa.

\section{Conclusiones}

El número de casos de Him fue bajo, condición que estuvo asociada al sexo. El patrón tipo i fue el más frecuente, dado que los primeros molares fueron los que presentaron la alteración en comparación con los incisivos. Así mismo, la condición de severidad leve fue la más frecuente, lo cual muestra un panorama en el que los casos, en su mayoría, están afectados desde el punto de vista estético. No obstante, se recomienda hacer un seguimiento estrecho de los niños con HIM, con el fin de evaluar y evitar el desgaste rápido y la pérdida del esmalte, la hipersensibilidad, el incremento de la susceptibilidad hacia la caries e incluso la pérdida dentaria en los casos de mayor severidad.

\section{Agradecimientos}

Al Programa de Servicio Social de la Carrera de Cirujano Dentista de la fes Zaragoza por el apoyo asignado. A la M. S. P. Arcelia Meléndez Ocampo por sus contribuciones metodológicas. A los escolares, los padres de familia y las autoridades del plantel de instrucción primaria por las facilidades brindadas para la ejecución del proyecto. A la Línea de Investigación en Oclusión Dental de la fes Zaragoza, UNAM, clave LI-FESZ-230506, por el apoyo y el aval del proyecto y el trabajo de investigación.

\section{Referencias}

[1] Weerheijm KL, Duggal M, Mejàre I, Papagiannoulis L, Koch G, Martens L, et al. Judgement criteria for molar incisor hypomineralisation ( $\mathrm{MIH})$ in epidemiologic studies: a summary of the European meeting on $\mathrm{MIH}$ held in Athens, 2003. Eur J Paediatr Dent. 2003;4:110-3.

[2] Segovia VA, Estrella RR, Medina-Solís CE, Maupomé G. Severidad de caries y factores asociados en preescolares de 3-6 años de edad en Campeche, México. Rev Salud Pública. 2004;7:56-69.

[3] Vallejos-Sánchez AA, Medina-Solís CE, CasanovaRosado JF, Maupomé G, Casanova-Rosado AJ, Minaya-Sánchez M. Enamel defects, caries in primary dentition and fluoride sources: relationship with caries in permanent teeth. Gac Sanit. 2007;21:227-34.

[4] Kuscu OO, Sandalli N, Dikmen S, Ersoy O, Tatar I, Turkmen I, et al. Association of amoxicillin use and molar incisor hypomineralization: visual and mineral density evaluation. Arch Oral Biol. 2013;58:1422-33.

[5] Phipps KR. No evidence to support the claim that amoxicillin causes molar-incisor hypomineralization. J Evid Based Dent Pract. 2012;12:73-5.

[6] Laisi S, Ess A, Sahlberg C, Arvio P, Lukinmaa PL, Alaluusua S. Amoxicillin may cause molar incisor hypomineralization. J Dent Res. 2009;88:132-6.

[7] Ciarrocchi I, Masci C, Spadaro A, Caramia G, Monaco A. Dental enamel, fluorosis and amoxicillin. Pediatr Med Chir. 2012;34:148-54.

[8] Tariq A, Alam Ansari M, Owais Ismail M, Memon Z. Association of the use of bacterial cell wall synthesis 
inhibitor drugs in early childhood with the developmental defects of enamel. Pak J Med Sci. 2014;30:393-7.

[9] Souza JF, Costa-Silva CM, Jeremias F, Santos-Pinto L, Zuanon AC, Cordeiro RC. Molar incisor hypomineralisation: possible aetiological factors in children from urban and rural areas. Eur Arch Paediatr Dent. 2012;13:164-70.

[10] Elfrink ME, Moll HA, Kiefte-de Jong JC, El Marroun $\mathrm{H}$, Jaddoe VW, Hofman A, et al. Is maternal use of medicines during pregnancy associated with deciduous molar hypomineralisation in the offspring? A prospective, population-based study. Drug Saf. 2013;36:627-33.

[11] Weerheijm KL. Molar incisor hypomineralisation (мIH). Eur J Paediatr Dent. 2003;4:114-20.

[12] Kellerhoff NM, Lussi A. Molar-incisor hypomineralization. Schweiz Monatsschr Zahnmed. 2004;114:243-53.

[13] Alaluusua S. Aetiology of molar-incisor hypomineralisation: a systematic review. Eur Arch Paediatr Dent. 2010;11:53-8.

[14] Gotler M, Ratson T. Molar incisor hypomineralization (МIH). A literature review. Refuat Hapeh Vehashinayim. 2010;27:10-8.

[15] Janković S, Ivanović M, Davidović B, Lecić J. Distribution and characteristics of molar-incisor hypomineralization. Vojnosanit Pregl. 2014;71:730-4.

[16] Cole E, Ray-Chaudhuri A. Molar-incisor hypomineralization (MIH). Dent Update. 2012;39:222-4.

[17] Grošelj M, Jan J. Molar incisor hypomineralisation and dental caries among children in Slovenia. Eur J Paediatr Dent. 2013;14:241-5.

[18] Da Costa-Silva CM, Jeremias F, DeSouza JF, Cordeiro R de C, Santos-Pinto L, Zuanon AC. Molar incisor hypomineralization: prevalence, severity and clinical consequences in Brazilian children. Int J Paediatr Dent. 2010;20:426-34.

[19] Bhaskar SA, Hegde S. Molar-incisor hypomineralization: prevalence, severity and clinical characteristics in 8- to 13-year-old children of Udaipur, India. J Indian Soc Pedod Prev Dent. 2014;32:322-9.

[20] Da Costa-Silva CM, Ambrosano GM, Jeremias F, De Souza JF, Mialhe FL. Increase in severity of molar-incisor hypomineralization and its relationship with the colour of enamel opacity: a prospective cohort study. Int J Paediatr Dent. 2011;21:333-41.

[21] Jans MA, Díaz MJ, Vergara GC, Zaror SC. Prevalencia y severidad de la hipomineralización molar incisal en pacientes atendidos en las clínicas odontológicas de la Universidad de La Frontera, Chile. Int J Odontostomat. 2011;5:133-40.
[22] Mathu-Muju K, Wright JT. Diagnosis and treatment of molar incisor hypomineralization. Compendium. 2006;27:604-11.

[23] Pitiphat W, Luangchaichaweng S, Pungchanchaikul P, Angwaravong O, Chansamak N. Factors associated with molar incisor hypomineralization in Thai children. Eur J Oral Sci. 2014;122:265-70.

[24] Wuollet E, Laisi S, Salmela E, Ess A, Alaluusua S. Background factors of molar-incisor hypomineralization in a group of Finnish children. Acta Odontol Scand. 2014;72:963-9.

[25] Petrou MA, Giraki M, Bissar AR, Basner R, Wempe C, Altarabulsi MB, et al. Prevalence of molar-incisor-hypomineralisation among school children in four German cities. Int J Paediatr Dent. 2014;24:434-40.

[26] Neg JJ, Eu OC, Nair R, Hong CH. Prevalence of molar incisor hypomineralization (MIH) in Singaporean children. Int J Paediatr Dent. 2014;24:286-92.

[27] Ghanim A, Morgan M, Mariño R, Bailey D, Manton D. Molar-incisor hypomineralisation: prevalence and defect characteristics in Iraqi children. Int J Paediatr Dent. 2011;21:413-21.

[28] Parikh DR, Ganesh M, Bhaskar V. Prevalence and characteristics of molar incisor hypomineralisation (MIH) in the child population residing in Gandhinagar, Gujarat, India. Eur Arch Paediatr Dent. 2012;13:21-6.

[29] Mahoney EK, Morrison DG. Further examination of the prevalence of $\mathrm{MIH}$ in the Wellington region. N Z Dent J 2011;107:79-84.

[30] Brogardh S, Matsson L, Klingberg G. Molar-incisor hypomineralization and oral hygiene in 10-to-12year-old Swedish children born preterm. Eur J Oral Sci. 2011;119:33-9.

[31] Kar S, Sarkar S, Mukherjee A. Prevalence and distribution of developmental defects of enamel in the primary dentition of IVF children of West Bengal. J Clin Diagn Res. 2014;8:73-6.

[32] Allazzam SM, Alaki SM, El Meligy OA. Molar incisor hypomineralization, prevalence, and etiology. Int J Dent. 2014;2014:234-508.

[33] Condò R, Perugia C, Maturo P, Docimo R. мıн: epidemiologic clinic study in paediatric patient. Oral Implantology. 2012;5:58-69.

[34] López Jordi MDC, Cortese SG, Álvarez L, Salveraglio I, Ortolani AM, Biondi AM. Comparison of the prevalence of molar incisor hypomineralization among children with different health care coverage in the cities of Buenos Aires (Argentina) and Montevideo (Uruguay). Salud Colect. 2014;10:243-51.

[35] Álvarez L, Hermida L. Hipomineralización molar -incisiva (MIH): una patología emergente. Odontoestomatol. 2009;11:4-11. 